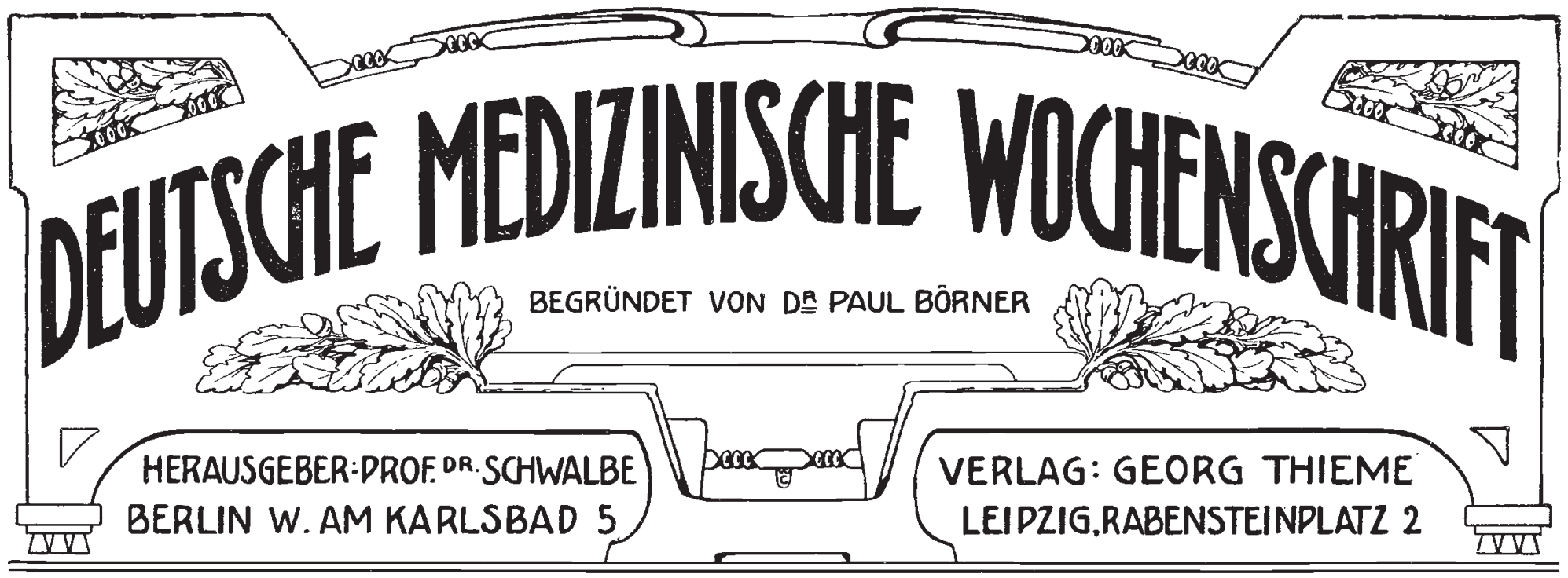

№ᅳ 16

BERLIN, DEN 22. APRIL 1909

35. JAHRGANG.

\section{Ueber Einklemmung bzw. Strangulation der Cauda equina.}

Von H. Oppenheim und F. Krause in Berlin.

Wir hatten vor einer Reihe von Jahren Gelegenheit, einen Patienten $z u$ beobachten und $z u$ behandeln, der unter den Erscheinungen einer Kompression der Cauda equina erkrankt war. Man hätte an einen Tumor denken können, wenn nicht die Symptome auffallend großen Schwankungen ausgesetzt gewesen wären; speziell galt das für die Sehnenphänomene, die bald fehlten, bald deutlich auslösbar waren. Lues konnte ausgeschlossen werden. War auch bei Sarkomatose schon ähnliches beobachtet worden, so schien dieser Annahme das Gesamtbild nicht zu entsprechen. Die Schwere der Erscheinungen, besonders die Intensität der Schmerzen, drängte schließlich zur operativen Behandlung.

Bei dieser bot sich uns ein überraschendes Bild. In der Höhe des vierten Lumbalwirbels zeigte sich der Wirbelkanal verengt (Fig. 1), die Verengung ging vom Knochen selbst aus, ohne daß

$$
\text { Fig. } 1 .
$$



ein kariöser oder geschwulstiger Proze $\beta$ an diesem entdeckt werden konnte. Die Wurzeln der Cauda waren in den knöchernen Engpaß wie eingekeilt, dagegen oberhalb desselben auseinandergedrängt (Fig. 2) - so etwa wie ein Bündel Wollfäden, das bei Annäherung des verschieblichen oberen an den fixierten unteren Pol auseinanderweicht -, sodaß $\beta$ diese Wurzeln bei der Eröffnung der Dura mater prolabierten. Außerdem fiel uns die starke Vermehrung des
Liquor cerebrospinalis auf, der bei Eröffnung der Dura mater aus den Subarachnoidealräumen oberhalb des Engpasses hervorstürzte.

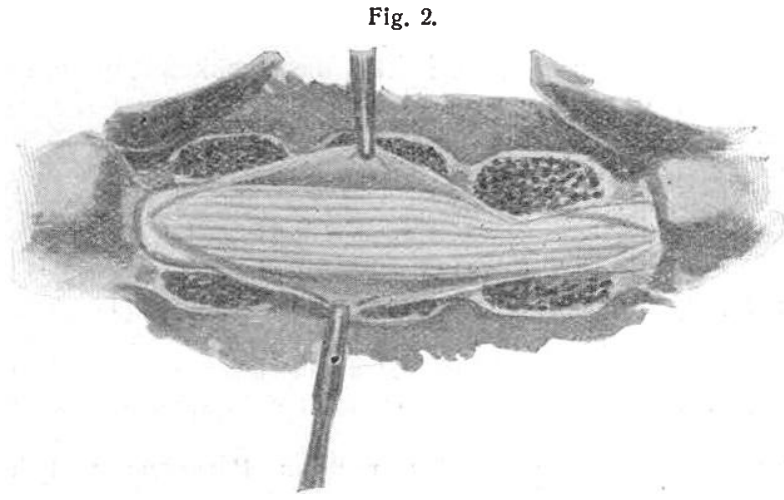

Es trat zunächst eine Besserung ein, dann aber erfolgte der Exitus, dem sehr heftige Reizerscheinungen vorausgingen.

Der Fall, auf den der eine ${ }^{1}$ ) von uns schon einmal kurz hingewiesen hat, hat uns viel zu denken gegeben, ist aber unaufgeklärt geblieben, da die Obduktion verweigert wurde.

Eine neue gemeinschaftliche Beobachtung gibt uns nun Anlaß, auf ihn zurückzukommen.

G. H., 43jähriger Kaufmann aus Prag, hatte, wie er angibt, schon in den Jahren 1894/95 nach längeren Märschen zuweilen über Schwere und Müdigkeit in den Beinen, namentlich im rechten zu klagen, seltener über ein Gefühl von "Stumpfheit" in der Wadengegend. Das ereignete sich bis zum Jahre 1903 kaum ein- bis zweimal im Monat. Eine im Jahre 1896 von Prof. W. vorgenommene Untersuchung ergab keinerlei Veränderungen am Nervenapparat, soda $\beta$ von diesem Arzt die bestehenden Krampfadern als Ursache der Beschwerden beschuldigt wurden. Im Sommer 1906 stellten sich schwerere Störungen ein. Etwa acht Tage nach einer Ueberanstrengung und Erkältung schnappte ich bei einer plotzlichen Bewegung zusammen und muBte, da mir jede Bewegung Schmerzen verursachte, das Zimmer hüten". Es bestanden hauptsiichlich Schmerzen im Kreuz, Ziehen und Schmerzen in den Beinen, namentlich beim Gehen. H. hielt die Beschwerden, wegen deren er zwei Tage im Bett blieb, für rheumatische. Die Schmerzen kamen von Zeit zu Zeit, fast immer als Müdigkeitsfolgen, und schwanden oft nach halbminutenlangem Sitzen. Einen ähnlichen Anfall von drei Tagen Dauer hatte Patient Anfang März 1908. Nach derartigen Attacken mußte er eine Zeitlang - bis zu zwei Wochen - sehr vorsichtig beim Gehen sein, um die Schmerzen zu verhüten. Im Februar 1908 wurde als Ursache der Beschwerden von Prof. E. der Druck von Varizen auf die Nervenstränge beschuldigt. Krampfaderoperation wurde emp. fohlen. Dann folgte eine Periode des Wohlbefindens, in der Patient sich viel und frei bewegen konnte, ohne Schmerzen zu verspüren. $\mathrm{Da}$ passierte am 30. Oktober 1908 um 101/2 Uhr vormittags folgendes: Patient ging über die Friedrichstrasse in Berlin, er sah einen Mann schwanken und suchte ihn vor dem Fallen zu bewah. ren, indem er ihn mit den Armen auffing und stützte; er mußte viel Kraft dabei aufwenden, sich bücken und wurde noch ein paar

1) H. Oppenheim, Zur Symptomatologie und Therapie der sich im Umkreis des Rückenmarks entwickelnden Neubildungen. Mitteilungen aus den Grenzgebieten 1906, Bd. 15, H. 5. S. hier auch die Abbildung. 
Schritte mit fortgeschleppt. Sofort verspürte er einen Schmerz in der linken Lendengegend, dieser steigerte sich von Minute zu Minute bis zil der Heftigkeit, daß der Kranke sich nach einer - viertel bis halben Stunde nicht mehr aufrecht erhalten konnte und zu Bett gebracht werden mußte. Es handelte sich besonders um einen sehr heftigen Rürkenschmerz bei jedem Versuch, die Lage zu wechseln, um heftiges Ziehen in den Beinen, die fast völlig gelähmt schienen, und um fast völlige Unempfindlichkeit des Gesäßes.

Am nächstfolgenden Abend hatte der pine von uns (Oppenheim) Gelegenheit, den Patienten im Hotel zu untersuchen. Er nahm im Bett die Rückenlage ein, die er ängstlich festhielt und aus der er sich nur unter heftigen Kreuz- und Beinschmerzen sehr langsam herausbringen konnte. Es fanden sich außer den starken Reizerscheinungen erhebliche Ausfallssymptome im Bereich des Plexus lumbosacralis beiderseits. Patient stand so unter dem Eindruck des aknten Ereignisses, da 3 er nur anf dieses hinwies. Und so stellte Oppenheim die Diagnose einer traumatischen Hämorrhagie in den Canalis vertebralis oder sacralis im Bereich der Canda, empfahl absolute Ruhe, Blutentziehung etc. sowie Ueberführung in ein Sanatorium. Am 1. November 1908 wurde Patient ins Hansasanatorium aufgenommen.

Die am 4. November erneuerte Untersuchung ergab folgendes: Kniephänomen rechts vorhanden, links fehlend, Fersenphänomen in der Rückenlage beiderseits nicht zu erzielen. Das rechte Bein wird von der Unterlage nur etwa 1/2 Fuß hoch erhoben, Beugung und Streckung des Unterschenkels ziemlich vollkommen, FuBstreckung nur in Adduktion (M. tibialis anticus), Abduktion fehlt; Zehenbewegung fehlt fast vollkommen, ebenso die Plantarflektion des Fußes.

Das linke Bein hebt Patient bis zur halben Höhe mit etwas größerer Kraft als das rechte; Beugung und Streckung des Unterschenkels wie rechts; Abduktion des Fußes gelingt etwas unvollkommen; Beugung des Fußes ziemlich kräftig und Zehenbewegung nahezu rollkommen erhalten.

Starke Erschwerung des Harnens, auch Inkontinenz beim Husten. Beim Urinieren verspürt er Brennen und anderweitige Parästhesien in der Harnröhre. Der Sphinkter ani soll nicht funktionieren. Erektionen und Libido fehlen vollkommen, doch scheint die Angabe nicht ganz sicher.

Elektrisch im Gebiete des rechten Peroneus und Tibialis posticus ziemlich erhebliche quantitative Abnahme der Erregbarkeit, keine Entartungsreaktion.

Parästhesien stellen sich fast nur bei Bewegungen ein, und zwar in der rechten Wade, am Fußrücken und in der rechten Gesäßgegend.

Sensibilität für Berührungen und Nadelstiche am rechten Fußrücken und in der Planta pedis abgestumpft, auch in der Wadengegend besteht Hypalgesie. Deutliche taktile Anästhesie und völlige Analgesie in der rechten Glutäalgegend, desgleichen am Scrotum. Lagegeftihl an den Zehen erhalten. Keine Druckempfindlichkeit der Wirbelsäule und des Kreuzbeins, keine ödematösen Schwellungen.

Therapie: Jodkalium 5,0:150,0; später dazu Jothion-Inunktionen.

7. November. Auch die Beugung des linken Fußes wird nur mit geringer Kraft ausgeführt. Die Schmerzen verlegt Patient heute an die AuBenseite beider Unterschenkel. Bei tiefem Atmen hat er eine Druckempfindung in der linken Lendengegend. Das Urinieren soll leichter gehen, auch haben die Parästhesien nachgelassen.

Facialis und Hypoglossus frei, Pupillarreaktion prompt, Augenbewegungen normal. Ophthalmoskopisch normal. "Mein Husten geht mir bis in die Fußspitzen." Taubheitsgefühl in der Gesäßgegend und am Penis; soll schon vor dem Unfall einige Male anfallsweise für 20 Minuten aufgetreten sein.

Die Röntgenaufnahme (Dr. Co h n) ergibt einen Schatten zwischen Processus transversus des zweiten und dritten Lendenwirbels rechts.

9. November. Lumbalpunktion (Oppenheim) zwischen dem zweiten und dritten Lendenwirbel. Entnahme von etwa $1 \mathrm{ccm}$ Liquor, keine besondere Drucksteigerung, nur vereinzelte rote Blutkörperchen und Lymphozyten.

13. November. Motorische Kraft in beiden Beinen besser. Sensibilitätsstörung in der Gesäß- und Hodengegend geringer. Klagt über Schmerzen an der Außenseite des linken Unterschenkels, weniger rechts.

16. November. Patient meint, daß sowohl in bezug auf die Schmerzen wie auf die Gefühllosigkeit in der GesäBgegend eine Besserung eingetreten sei und daB die Harnentleerung leichter vonstatten gehe. Verhalten der Sehnenphänomene unverändert. Das rechte Bein hebt er jetzt über zwei Fuß hoch von der Unterlage und mit ziemlich guter Kraft; Beweglichkeit im Kniegelenk gut, Streckung des Fußes ziemlich gut, aber es fehlt die Wirkung der Abduktoren. Plantarflektion des Fußes fast gleich null. Zehenbeweglichkeit an scheinend etwas besser als bei letzter Untersuchung. Patient kann den rechten Oberschenkel etwas auswärts- und kraftvoll einwärts- rollen. Das linke Bein hat im ganzen etwas Vorspruyg vor dem rechten in bezug auf die motorische Kraft. Viel besser sind hier die Zehenbewegungen.

Pinselberührungen werden an der Vorderfläche der Beine iiberall gefühlt, Nadelstiche ebenfalls, speziell auch am Fußrücken und in der Planta rechts. Auch werden Pinselberührungen in der rechten Glntäalgegend gefühlt, wenn auch in den zilkumanalen Partien undeutlich, sehr ansgesprochen ist dagegen die Analgesie dieser Gegend. Füussigen Stuhl und Flatus kann Patient nicht halten. Die mechanische Muskelerregbarkeit ist an beiden Unterschenkeln etwas erhöht. Temperatursinn verhält sich wie Schmerzgefühl.

18. November. Wassermannsche Reaktion negativ.

2. Dezember. Kniephänomen fehlt links, ist rechts vorhanden. Das rechte Bein wird in der Hüfte etwa drei Fuß erhoben. mit ziemlich guter Kraft, Einwärtsrollung gut, Auswärtsrollung mit verminderter Kraft. Bewegung im rechten Kniegelenk kraftroll, im Fußgelenk wirkt fast nur der M. tibialis anticus mit ziemlich guter Kraft, Plantarflektion fehlt. Die Zehen werden ziemlich ausgiebig gestreckt und gebeugt, aber ohne Kraft.

Links Bew'egungen im Hüft- und Kniegelenk wie rechts. Bei der Dorsalflektion des Fußes beteiligen sich alle Muskeln, am wenigsten die Peronei; im ganzen ist die Bewegung ausgiebiger als rechts. Auch die Plantarflektion des Fußes gelingt hier, aber mit etwas verminderter $\mathrm{K}$ raft, Zehenbewegungen ausgiebiger und etwas kräftiger als rechts. Patient hat noch mannigfaltige Parästhesien und Schmerzen, letztere aber weniger als früher, sie hindern ihn weniger an seinen Bewegungen. Pinselber ührungen werden an beiden Beinen überall gefühlt, auch Nadelstiche im ganzen gefühlt, in der rechten Planta pedis weniger als links. Glutäalmuskulatur beiderseits sehr schwach, rechts noch etwas atrophischer als links.

In der rechten Glutäalgegend besteht noch taktile Anästhesie, aber in geringerer Ausdehnung (als anfangs), die Analgesie nimmt noch ein ziemlich großes Feld ein, besteht aber in völliger Intensität nur in der Nachbarschaft der Rima ani, um sich von da nach außen mehr abzuschwächen. Nachdem Patient bei der Untersuchung einige Zeit auf dem Bauch und den Seiten gelegen hat, verspürt er heftige Schmerzen in den Waden.

Zur rechten des zweiten (oder dritten?) Lendenwirbels hat er bei mehrfach von ihm selbst vorgenommener Prüfung eine Druckempfindlichkeit mit Ausstrahlung nach der Glutäalgegend festgestellt.

17. Dezember. Schmerzen und Parästhesien nur bei Bewegungen und nicht heftig. Bei Druck auf die Gegend des zweiten oder dritten Lendenwirbel-Querfortsatzes (rechts) stellt sich ein Schmerz ein, der nach der Glutäalgegend ausstrahlt, auch spontan bei Bewegungen und bestimmten Lagen. Perkutorisch hier nichts $\mathrm{Ab}$ normes.

Glutäalmuskulatur beiderseits schlaff, rechts mehr als links. Die aktive Anspannung sehr gering, besonders auf der rechten Seite. Verhalten der Sehnenreflexe wie früher. Die Incontinentia urinae et alvi soll nicht mehr bestehen (?), auch die Erschwerung des Harnens nicht erheblich sein.

Die aktive Beweglichkeit im ganzen wie bei der letzten Untersuchung, vielleicht etwas kräftiger, aber es sind keine neuen Bewegungen hinzugekommen. Sensibilităt an den Fußen wie bisher.

Im Gebiet des rechten $N$. peronens und tibialis posticus Entartungsreaktion, an der nur der M. tibialis anticus nicht teilnimmt; links ungefăhr dasselbe, nur sind die Veränderungen der elektrischen Erregbarkeit hier nicht so ausgesprochen, so ist die direkte faradische Erregbarkeit im rechten Peronealgebiet (mit AusschluB des Tibialis anticus) ganz erloschen, während sie links noch spurweise erhalten ist; auch ist die direkte faradische Erregbarkeit in der linkseitigen Wadenmuskulatur besser erhalten wie rechts. An der ganzen Hinterfläche des rechten Oberschenkels, speziell in den mittleren Bezirken, ist das Gefühl für Schmerz und Berührungen herabgesetzt; in demselben Gebiet Thermanästhesie. Im linken Extensor cruris quadriceps besteht eine entschiedene Schwäche. Erektion und Pollution sollen unter Libido im Schlaf stattgefunden haben.

Nunmehr schien die operative Behandlung angezeigt, da die schweren Veränderungen der elektrischen Erregbarkeit, die Ausbreitung der Schwäche auf den linken Quadriceps, der Anästhesie auf die Hinterfläche des rechten Oberschenkels eine gewisse Progredienz des Leidens erkennen ließen. Dem Operateur wurde folgender Bericht (Oppenheims) vorgelegt: „Bei dem p. H. haben sich seit einigen Jahren allmählich bzw. in Schüben die Erscheinungen eines Leidens vorwiegend im Gebiete der Sakralwurzeln, mehr der rechten Seite entwickelt, doch ist wohl auch noch die fünfte Lumbalis und auf der linken Seite außerdem die vierte beteiligt. Die Erscheinungen bestehen 
in Schmerzen, die ihren Sitz in der Lenden-Gesäßgegend sowie im Gebiet der Ischiadici haben, in Parästhesien ungefähr von derselben Verbreitung, in atrophischer Lähmung, die rechts das Gebiet des Peroneus und Tibialis posticus fast völlig betrifft mit Verschonung des M. tibialis anticus, links dasselbe Gebiet in geringerem Maße, außerdem hier noch den Extensor cruris quadriceps unvollkommen, ferner die Glutaei vorwiegend rechts. Dazu kommen Sensibilitätsstörungen, am ausgesprochensten im Gebiet der dritten und vierten Sacralis rechts (circumanal), im geringen Maße im Bereich der oberen Sakralwurzeln, leichte Störungen der Blasen- und Mastdarmfunktion.

Der Prozeß könnte vom Lumbosakralmark (Conus und Epiconus) oder von der Cauda equina ausgehen. Die Asymmetrie, die Schmerzhaftigkeit, die geringe Betonung der Sphinkterenstörung, das Freibleiben der oberen Lendenwurzeln macht mir die Annahme einer vom Rückenmark ausgehenden Erkrankung unwahrscheinlich. Ich nehme vielmehr an, daß die Erkrankung am oberen Bereich der Cauda equina in der Höhe des dritten Lendenwirbels und vorwiegend rechts sitzt; freilich könnte sie anch nach oben und selbst nach unten etwas über dieses Terrain hinausgreifen. Da nichts für Syphilis spricht (auch Wasser mann negativ), ist an eine Neubildung bzw. einen ihr durch dio Kompressionswirkung verwandten Prozeb (Gefäßgeschwulst?, Meningitis serosa?) zu denken.

Bei der Unwirksamkeit der internen Therapie bin ich für die Laminektomie, zunächst in der Höhe des dritten Lendenwirbels." Prof. H. Oppenheim.

Auf Grund gemeinschaftlicher Untersuchung und Erwägung wurde dann die Operation beschlossen und am 23. Dezember ausgeführt.

In linker Seitenlage wird durch einen Längsschnitt der zweite, dritte und vierte Lendendornfortsatz freigelegt. Nach Entfernung des zweiten und dritten Bogens liegt die Dura in $6 \mathrm{~cm}$ Ausdehnung frei, pulsiert garnicht und zeigt siclı in den oberen zwei Dritteln durch eine Ansammlung von Flüssigkeit gespannt, während unten die Spannung durch eine solide Masse bedingt zu sein scheint. Bei Eröffnung der Dura stïrzt Liquor hervor und zugleich damit die zusammengeballten Wurzeln der Cauda equina (Fig. 3). Nach Spal-

Fig. 3.

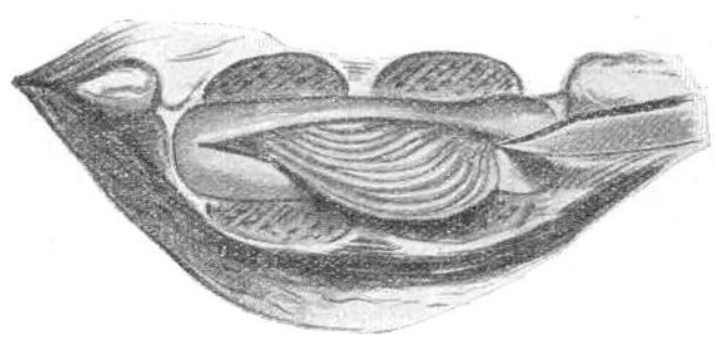

tung der Dura in der ganzen Wundlänge und nach Emporheben der Cauda equina mit einem stumpfen Haken kommt im unteren Wundbezirk ein doppeltbohnengroßer, längsstehender, flach gewölbter Tumor zum Vorschein (Fig. 4), der von der unveränderten Dura

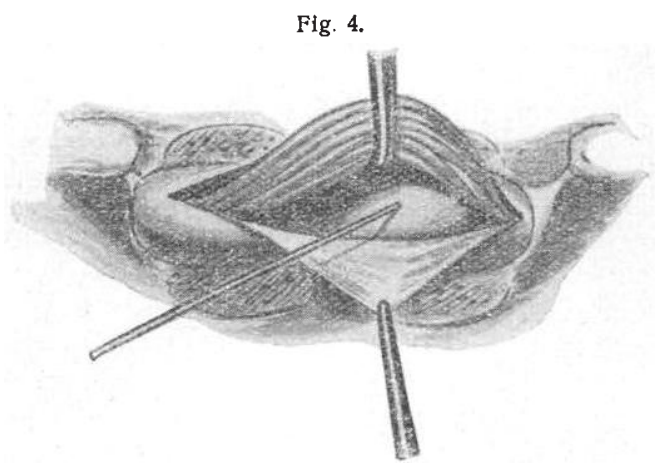

der hinteren Fläche der W'irbelkörper überzogen ist. Dem Gefühl nach ișt dieser Tumor fast weich. Wegen der Möglichkeit einer Bilutgeschwulst wird erst punktiert und aspiriert. Da kein Blut kommt, wird eine Längsinzision durch die vordere Dura über der ganzen Höhe der Geschwulst ausgeführt und diese mit dem scharfen Löffel möglichst radikal entfernt. Die Sondierung ergibt ein nach unten auf Fingerlänge, nach oben mindestens im Gebiet der nächsten zwei Wirbelbogen freies Terrain. Naht der Dura und der Wunde.
24 Dezember. Freies Sensorium, keine wesentlichen Beschwerden, keinerlei neue Ausfallserscheinungen im Bereich der Lumbosakralwurzeln. Verhalten der Sehnenphänomene wie vorher. Die Plantarflektion wird am linken $\mathrm{FuB}$ in voller Ausdehnung ausgeführt, und die Kraft ist dabei anscheinend erheblicher als vor der Operation.

Rechts kontrahiert sich der M. tibialis anticus mit voller Kraft, auch ist eine leichte Plantarflektion ausführbar.

Sensibilität an der Planta pedis und der Zirkumanalgegend wie vorher.

26. Dezember. Urinentleerung zweimal spontan Erektionen.

31. Dezember. Patient klagt, daß die Harnentleerung schwerer als vorher und mit einem schmerzhaften Priapismus verbunden sei, Spannungsgefühl in der Wurzel des Penis.

Er schildert eine Art von Incontinentia alvi, die aber in der Tat nicht besteht, er will nicht die genügende Herrschaft über den SchlieBmuskel haben. Schmerzen bei Bewegungen nur im Kreuz und an der Operationsstelle.

Parästhesien gänzlich geschwunden.

Im rechten Bein Muskeltonus herabgesetzt, besonders im Fußgelenk, Kniephänomen rechts lebhaft, fehlt links; Fersenphänomen fehlt in Rückenlage beiderseits.

Die aktive Beweglichkeit im Hüft- und Kniegelenk im ganzen erhalten, nur Hüftbeugung in beschränkter Ausdehnung wegen des dabei eintretenden Wundschmerzes.

Rechts Dorsalflektion des Fußes nur durch den M. tibialis anticus, der sich dabei kräftig anspannt. Plantarflektion fehlt. Zehenbeugung und -streckung gelingt, aber kraftlos. Links Bewegungen im Hüftgelenk in nahezu voller Ausdehnung, aber mit_etwas verringerter Kraft. Kniegelenk im ganzen normal beweglich. Bei der Streckung des Fußes spannen sich zwar alle Muskeln an, aber die Aduluktoren mehr als die Abduktoren. Plantarflexion des Fußes mit nahezu völliger Kraft; Zehenbewegungen ausgiebiger und kräftiger als rechts. Patient macht darauf aufmerksam, daß er jetzt die vierte und fünfte Zehe des linken Fußes bewegen könne, was vor der Operation nicht möglich gewesen sei.

Am linken Fuß Sensibilität für alle Reize normal.

Am rechten FuB werden Berührungen und Druck überall unterschieden, auch fühlt Patient Nadelstiche in der Planta pedis lebhaft. Temperatursinn erhalten. An der rechten Glutäalgegend in einer handbreiten Zone Anästhesie und Analgesie sowie Thermanästhesie, die anscheinend etwas an Ausdehnung gewonnen hat (?); diese Zone erstreckt sich auch auf die Hinterflache des Oberschenkels, an w'elcher jedoch die Intensität der Sensibilitätsstörung nicht so groß ist.

Der reflektorishe SchluB des Sphincter ani bei Einführung eines Fingers ist nicht kräftig, aber auch nicht aufgehoben. Patient verspürt Schmerz bei der Untersuchung.

Keine meningitischen Symptome. Einzelne abendliche Temperatursteigerungen am 7 . bis 2 . Tage nach der Operation waren durch einen Blasenkatarrh bedingt, sie verschwanden nach regelmäßiger Spülung mit Bor- und Höllensteinlösungen.

Die mikroskopische Untersuchung der exstirpierten Geschwulstbestandteile (Prof. Oesterreich und Dr. Heymann) ergab im wesentlichen Knorpelgewebe mit spärlichen Knorpelzellen. Es war mit Fasern ziemlich kräftig durchsetzt; Blutgefäße waren außerordentlich spärlich, und nur an einigen Stellen fanden sich Anhäufungen von Granulationszellen. Keine Nekrosen, spärlich verkalkte Stellen, kein Knochengewebe.

20. Januar 1909. Schmerzen im rechten Bein ganz geschwunden. Patient betont besonders, daB die Schmerzen und Parästhesien, die früher beim Niesen und Husten auftraten, völlig geschwunden sind.

10. Februar. Die Motilität des rechten Beines zeigt ungefähr die früheren Verhältnisse, im linken sind die Oberschenkelbewegungen nahezu normal, auch die des Unterschenkels; die FuBstreckung ist ausgiebig und ziemlich kräftig, doch mit überwiegender Adduktion; Plantarflektion ziemlich kräftig; die Zehenbewegungen haben an Ausgiebigkeit gewonnen. In der rechten Planta pedis werden Berührungen teilweise nicht gefühlt, Nadelstiche abgeschwächt.

In der rechten Glutäaloberschenkelgegend hat sich die Gefühlsstörung gebessert, indem das Gebiet der völligen Anästhesie ein kleineres geworden ist. Patient macht darauf aufmerksam, daß er wieder mit voller Kraft husten und niesen kann, ohne Schmerzen, eine Besserung, die sich unmittelbar nach der Operation gezeigt habe.

Im Laufe der letzten Wochen ist es zweimal zu Pollutionen gekommen. Einige Male soll sich beim kräftigen Husten und Niesen etwas Urin entleert haben.

Patient macht jetzt Gehversuche bei doppelseitiger Unterstützung, auch kann er allein stehen.

27. Februar. Kraft in den Rotatoren des Oberschenkels und 
im linken Quadriceps jetzt normal, sonst bezüglich Motilität status idem. Patient geht jetzt mit Stock in der rechten Hand und leichter Unterstützung am linken Arm durchs Zimmer. Stuhlentleerung normal, keine Inkontinenz (auch nicht für weichen Stuhl und Flatus); Harnentleerung noch etwas erschwert, mit Bauchpresse, absatzweise, keine Inkontinenz. Sensibilität an den Füßen normal, in der Glutäaloberschenkelgegend unverändert. Glutäalmuskeln schwach.

Wenn wir die Krankengeschichte kurz zusammenfassen, haben wir folgenden Tatbestand: Bei einem sonst gesunden, nicht syphilitischen Manne, bei dem seit einer Reihe von Jahren zeitweise Schmerzen und Parästhesien sowie eine gewisse Beweglichkeitsbehinderung vorübergehend, namentlich nach Anstrengung, nach brüsken Bewegungen auftritt, stellt sich im Anschluß an einen Akt, bei dem er sich bücken und eine verhältnismäßig erhebliche Kraft aufwenden mußte (Stützen einer fallenden Person bzw. Heben und Halten einer Last) sofort ein Lenden-Kreuzschmerz und gleich darauf eine Lähmung in den Beinen ein, welche sich im Gebiet der Sakralund unteren Lumbalwurzeln lokalisiert. Es ist der Symptomenkomplex einer hochsitzenden Cauda-equina-Affektion, den der Kranke bietet, dabei sind die Bewegungen der unteren Rumpfgegend sehr schmerzhaft und ebenso wie alle exspira. torisch-pressorischen Akte mit Schmerzen und Parästhesien verknüpft („der Husten geht bis in die Fußspitzen“ etc.). Ein Teil der Beschwerden, namentlich der Reizerscheinungen, geht allmählich unter Jodtherapie etwas zurück. Dann ist der Verlauf ein schwankender, aber es tritt allmählich deutlicher der degenerative Charakter (EaR.) der Lähmung zutage, auch greift sie auf einen Muskel über, del anfangs verschont war (Extensor cruris, quadriceps sinister). Die Anästhesie zeigt $\mathrm{zwar}$ an einigen Stellen ein schwankendes Verhalten, dehnt sich aber an anderen (Hinterfläche des Oberschenkels) weiter aus. Unter diesen Verhältnissen gewann die Annahme eines raumbeschränkend wirkenden Prozesses, einer Neubildung, mehr und mehr an Boden. Die Erscheinungen von seiten des Nervensystems wiesen auf den dritten Lendenwirbel, dem entsprach auch eine druckempfindliche Stelle und wohl auch der Röntgenbefund (?). Bei der an dieser Stelle ausgeführten Laminektomie zeigt sich der Wirbelkanal beträchtlich verengt durch eine vom Körper des dritten Lendenwirbels ausgehende tumorartige Schwellung, die nach der mikroskopischen Untersuchung wohl als Enchondrom angesprochen werden muß. Oberhalb dieses Engpasses ist die Dura durch Liquor sowie durch die auseinanderstrebenden Wurzeln der Cauda aufgetrieben, sodaß bei ihrer Eröffnung nicht nur der Liquor hervorstürzt, sondern auch die (graurötlich verfärbten) Wurzeln prolabieren. Der Tumor wird, soweit er erkennbar, exstirpiert, die Verengung scheint damit beseitigt. Die Operation hat den Erfolg, daß die Schmerzen und Parästhesien selbst beim Husten, Niesen etc. sofort aufhören, daß auch in bezug auf einen Teil der Ausfallserscheinungen eine Besserung eintritt Längere Zeit, wenigstens zwei Wochen lang, wird die Besserung durch ein von einem Blasenkatarrh ausgehendes Fieber aufgehalten, dann aber gelangt der Kranke so weit, Steh- und Gehversuche zu machen. Ein Teil der Ausfallserscheinungen bleibt aber in unverminderter Intensität bestehen, und es läßt sich über die definitive Prognose noch nichts aussagen.

Der Fall könnte zu mancherlei epikritischen Betrachtungen Veranlassung geben. Wir wollen uns auf die Erörterung der Tatsachen beschränken, auf die der Titel unserer Abhandlung hinweist: Die Einklemmung der Cauda equina, diesen von uns nun zweimal beobachteten Vorgang.

Es handelt sich nicht um die einfache Kompression des Plerdeschweifes, sondern es spielen hier andere Momente eine Rolle. Es ist nicht die Symptomatologie einer allmählich erfolgenden Bedrängung der Wurzeln, sondern nach relativ unbedeutenden Vorboten ist es eine akut einsetzende Kompressionslähmung, die unter dem Einfluß einer gewaltsamen Muskelaktion zustande kommt. Das Bild, das die oporative Freilegung des entsprechenden Gebietes uns enthüllt, ist das der Zusammenschnürung und Abknickung der Caudawurzeln am Orte der Verengerüg und ihrer Auseinanderdrängung oberhalb derselben. Es ist das am besten zu verstehen unter der Annahme einer Verschiebung, welche dio Cauda bei der Beugung des Oberkörpers gegen den Knochen bzw. gegen den Knochentumor erfährt, derart, daß sie um ein Stück nach oben verschoben, dann unter dem Einfluß der pressorisch wirkenden Muskelanstrengung so gebläht (auseinandergetrieben) wird, daß sie nicht zurückschlüpfen kann, sondern daß die Wurzeln abgeknickt werden. Erleichtert wird dieser Vorgang wahrscheinlich durch die Erweiterung, welche die die Wurzeln begleitenden Venen erfahren. Ist nun einmal der Engpaß durch die Wurzeln verstopft, so kommt als ein weiteres, die Kompression wesentlich förderndes Moment die Ansammlung und wohl auch vermehrte Sekretion des Liquor (Meningitis serosa) oberhalb der verengten Stelle hinzu.

Mit dieser Deutung gewinnen wir ein volles Verständnis für das Krankheitsbild.

Gewiß sind weitere Erfahrungen erwünscht. Auch dürften experimentelle Untersuchungen geeignet sein, über das $\mathrm{Zu}$ standekommen dieses Vorganges und seine Symptomatologie weiteres Licht $\mathrm{zu}$ verbreiten. 